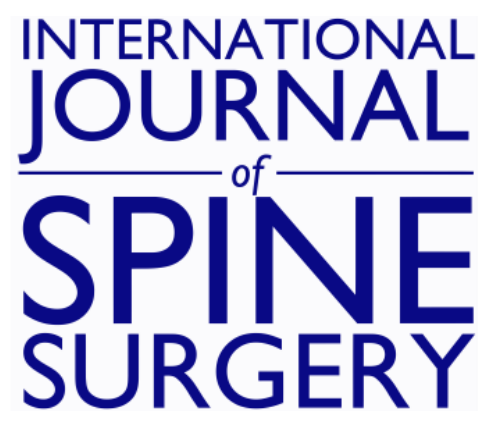

\title{
Readability Analysis of Patient-Accessible Information Regarding Ambulatory Surgical Center Procedures
}

Conor P. Lynch, Elliot D.K. Cha, Nathaniel W. Jenkins, James M. Parrish, Shruthi Mohan, Cara E. Geoghegan, Caroline N. Jadczak and Kern Singh

Int J Spine Surg 2021, 15 (5) 1046-1053

doi: https://doi.org/10.14444/8133

http://ijssurgery.com/content/15/5/1046

This information is current as of April 26, 2023.

Email Alerts Receive free email-alerts when new articles cite this article. Sign up at: http://ijssurgery.com/alerts 


\title{
Readability Analysis of Patient-Accessible Information Regarding Ambulatory Surgical Center Procedures
}

\author{
CONOR P. LYNCH, MS, ELLIOT D.K. CHA, MS, NATHANIEL W. JENKINS, MD, JAMES M. PARRISH, MD, \\ MPH, SHRUTHI MOHAN, BS, CARA E. GEOGHEGAN, BS, CAROLINE N. JADCZAK, BS, KERN SINGH, MD \\ Department of Orthopaedic Surgery, Rush University Medical Center, Chicago, Illinois
}

\begin{abstract}
Background: As spine surgery in the ambulatory setting becomes more frequent, patients should comprehend the difference from traditional hospital-based, outpatient settings. Limited research exists on the readability of online articles surrounding spine surgery in the ambulatory surgery center (ASC). In this study, we intend to evaluate the readability of online articles pertaining to spine surgery in the outpatient and ambulatory surgical settings.

Methods: Three search engines were queried, and the first 100 articles pertaining to each outpatient spine surgery search term were collected. Advertisements, videos, and peer-reviewed scientific articles were excluded. Articles were categorized by publishing source as follows: hospital or institution, general medical Websites, private practice, or surgery center. Flesch-Kincaid (FK) grade level, Flesch Reading Ease (FRE) score, word count, sentences per paragraph, words per sentence, and characters per word were evaluated for each article. Student's $t$ tests compared readability metrics between groups based on setting and procedure region.

Results: A total of 342 articles was analyzed; 279 articles were outpatient hospital related, and 63 ASC related. Flesch-Kincaid grade levels or FRE scores were not significantly different between outpatient hospital and ambulatory center. Comparison of ASC to outpatient articles from a hospital or institution source significantly differed in FRE score (40.7 versus 32.4) and FK grade level (12.3 versus 13.9; all $P<.05$ ). Articles addressing procedure type were significantly different in FRE score (36.2 versus 30.0) and FK grade level (13.0 \pm 2.1 versus $14.3 \pm 1.8)$.

Conclusions: Hospital, private practice, and medical journalists should be aware of significant differences in readability of patient-accessible ASC articles. These articles may be more difficult to read than outpatient hospital articles, and production of more reading-level-appropriate online literature is required.
\end{abstract}

Level of Evidence: 3

Clinical Relevance: There is a significant difference in the readability of patient-accessible ASC articles.

Other \& Special Categories

Keywords: ambulatory surgical center, outpatient spine surgery, patient education, readability

\section{INTRODUCTION}

While spine surgery was once synonymous with a lengthy inpatient stay, a growing number of spine procedures are now being performed on an outpatient basis. For procedures such as anterior cervical discectomy and fusion (ACDF), lumbar decompression (LD), and cervical laminectomy, the proportion of surgeries being performed in outpatient settings has increased substantially over the last 2 decades. ${ }^{1}$ In fact, for procedures such as LD, outpatient surgery may now be the rule rather than the exception. A recent review of the PearlDiver database revealed that outpatient ACDF procedures have doubled from 2011 to 2016. ${ }^{2}$ However, use of the term "outpatient" can include procedures performed both in outpatient hospital settings and in standalone ambulatory surgical centers (ASCs).

Since ASCs typically require patients to be discharged within 23 hours of their procedure and lack the capacity to deal with serious medical complications, careful selection of patients is required. As Chin et $\mathrm{al}^{3}$ noted, almost $80 \%$ of patients may be eligible candidates for spine surgery in an ASC. Common selection criteria for outpatient spine surgery include less complex operations (ie, primary, single-level procedures), ${ }^{4,5}$ lower body mass index and comorbidity burdens, ${ }^{3,5,6}$ as well as social factors such as living within 30 minutes of a hospital and having adequate assistance available at home. ${ }^{3}$ With appropriate patient selection in place, procedures in this setting can be quite safe. ${ }^{5,7} \mathrm{~A}$ recent systematic review by Sivaganesan et $\mathrm{al}^{8}$ found 
that less than $2 \%$ of ASC patients required transfer to a hospital after ACDF, posterior cervical fusion, and LD procedures and suggested that overall morbidity rates were comparable with inpatient procedures.

In addition to their safety, patients undergoing spine surgery in ASCs have demonstrated equivalent outcomes in terms of pain and disability, similar rates of narcotic consumption, and shorter lengths of stay compared with hospital-based procedures. ${ }^{4,5,7}$ Perhaps one of the most distinct benefits of outpatient settings for spine surgery is the decreased cost. Outpatient spine procedures can be associated with thousands of dollars in savings, ${ }^{8,9}$ with authors of 1 study of 1123 ACDF procedures demonstrating a $26 \%$ lower cost to commercial insurance for outpatient procedures than inpatient. ${ }^{10}$

While similar outcomes have been demonstrated between inpatient and outpatient settings for several spinal procedures, ${ }^{4,9,10}$ the experience of undergoing surgery in these settings may differ in several important ways. Differences in facility or hospital protocols as well as the emphasis on timely discharge in ASC settings can lead to different prescribing patterns for postoperative narcotic medications. ${ }^{4}$ Furthermore, patients undergoing surgery in ambulatory settings may expect their postoperative stays to be significantly shorter., ${ }^{4,11}$ While complication rates in outpatient settings are generally quite low, ${ }^{4,9}$ certain adverse events in an ambulatory setting may require transfer to a fully equipped hospital, and patients should be aware of this possibility.

Given the numerous benefits to both patients and institutions, it will be important for clinicians and patients to stay well informed about what outpatient spine surgery entails, as well as new developments or limitations. For patients undergoing ambulatory procedures, it may be important to obtain more information and at an earlier point during their clinical course. ${ }^{12}$ While direct consultation with a physician is almost always the best source of information for patients, the Internet provides a host of readily available resources that are both free and convenient for patients to access. However, when pursuing information about outpatient settings for their spine procedures, one significant concern that may arise is the readability of the information that is accessible to patients. In a national survey by the U.S. Department of Health and Human Services, researchers found that most US adults had an "intermediate" level of health literacy, ${ }^{13}$ and other organizations recommend a sixth to seventh grade reading level for healthrelated materials intended for patients. ${ }^{14-16}$ However, authors of several previous studies have demonstrated that online educational materials related to spine surgery that are accessible to patients tend to be written at a significantly higher reading level than those recommended and use a relatively high amount of jargon that may not be understood by many patients. ${ }^{17-20}$ Furthermore, material with more advanced reading levels may not actually be associated with higher quality of literature. ${ }^{21}$

When examining patient targeted literature, the source is important to consider. Even resources from top-rated orthopaedic institutions may provide literature well above what is recommended for easy patient comprehension and accessibility. ${ }^{18}$ Therefore, for emerging topics such as outpatient and ambulatory surgery, it raises the issue of whether differences exist between literature produced by hospitals offering procedures on an outpatient basis and by standalone ASCs. In this study, we seek to examine patient-accessible literature related to outpatient spine surgery and assess differences in readability based on publication source, procedure type, and whether the literature pertains specifically to hospital-based or ASC outpatient procedures.

\section{METHODS}

Institutional Review Board Approval: ORA \#14051301.

\section{Data Collection}

To collect appropriate articles for this study, searches were conducted using Google, Yahoo, and Bing search engines. The same 7 search strings ("outpatient spine surgery," "same day spine surgery," "surgery in an ambulatory surgical center," "outpatient lumbar fusion," "outpatient lumbar decompression surgery," "outpatient cervical surgery," and "outpatient cervical fusion") were used with each of the 3 search engines. The first 100 articles with relevance to each outpatient spine surgery search string were collected. Search results that included advertisements, videos, and peerreviewed scientific articles were excluded from this analysis. Additionally, articles pertaining to outpa- 
Table 1. Mean readability descriptive statistics.

\begin{tabular}{|c|c|c|c|c|}
\hline & Total $(n=342)$ & Outpatient $(n=279)$ & Ambulatory $(n=63)$ & $P$ Value $^{\mathrm{a}}$ \\
\hline Word count & $749.9 \pm 638.0$ & $731.6 \pm 624.2$ & $830.5 \pm 695.2$ & 0.267 \\
\hline Characters/word & $5.3 \pm 2.7$ & $5.3 \pm 3.0$ & $5.2 \pm 0.3$ & 0.719 \\
\hline Words/sentence & $19.7 \pm 4.2$ & $19.6 \pm 4.2$ & $20.5 \pm 4.1$ & 0.092 \\
\hline Sentences/paragraph & $2.8 \pm 1.9$ & $2.8 \pm 1.9$ & $2.7 \pm 1.9$ & 0.548 \\
\hline Flesch Reading Ease & $38.2 \pm 12.9$ & $38.8 \pm 13.1$ & $35.5 \pm 12.1$ & 0.073 \\
\hline Flesch-Kincaid grade & $12.7 \pm 2.7$ & $12.6 \pm 2.7$ & $13.1 \pm 2.6$ & 0.174 \\
\hline
\end{tabular}

${ }^{\mathrm{a}} P$ value calculated using independent $t$ tests (continuous).

tient surgery or ASCs were included for review, and any articles largely pertaining to the technical aspects of the procedure performed were excluded. All articles were reviewed by 2 individuals, and any disagreements were discussed until a consensus was reached.

\section{Article Classification and Subgroup Division}

Included articles were first classified based on whether they pertained to surgery in an outpatient hospital setting or an ASC setting. Included articles were additionally categorized according to publication source into 1 of 4 subgroups: hospital or institution (HI), general medical Websites (GMWs), private practice (PP), or surgery center (SC). Lastly, articles were further divided into 1 of 4 subgroups depending on which procedure the article was related: cervical, lumbar, combined, or not specified.

\section{Statistical Analysis}

Each included article was evaluated for readability using the following 6 metrics: Flesch-Kincaid (FK) grade level, Flesch Reading Ease (FRE) score, word count, sentences per paragraph, words per sentence, and characters per word (Table 1). Interpretation of the FRE score and associated FK grade level can be found in Table 2. Student's $t$ test was used to compare means for all 6 readability metrics between outpatient and ambulatory classifications. Average readability for each metric was then stratified separately by publication source and by procedure type. A Student's $t$ test was used to

Table 2. Flesch Reading Ease and Flesch-Kincaid grade scales.

\begin{tabular}{lll}
\hline $\begin{array}{l}\text { Flesch Reading } \\
\text { Ease Score }\end{array}$ & Flesch-Kincaid Grade & \multicolumn{1}{c}{ Notes } \\
\hline $100.0-90.0$ & Fifth grade & Very easy to read \\
$89.99-80.0$ & Sixth grade & Easy to read \\
$79.99-70.0$ & Seventh grade & Somewhat easy to read \\
$69.99-60.0$ & Eighth to ninth grade & Standard \\
$59.99-50.0$ & 10th to 12th grade & Fairly difficult to read \\
$49.99-30.0$ & College or university & Difficult to read \\
$29.99-0.0$ & College graduate & Very difficult to read \\
\hline
\end{tabular}

determine differences in means by publication source and procedure type (Table 3 ).

\section{RESULTS}

A total of 342 articles was included in the final analysis; 279 articles were classified as pertaining to outpatient hospital settings and 63 to ASC settings. No significant differences were observed in mean scores for any of the used readability metrics between outpatient hospital and ASC classifications (Table 1). No significant differences between article classifications were demonstrated for mean word count when articles were stratified by publication source (Table 3 ). When articles were stratified by procedure type, mean word count was significantly different between classifications for cervical $(P=$ .022), lumbar $(P=.024)$, and not specified articles $(P$ $=.012)$. No significant differences in mean characters per word between classifications were demonstrated for any publication source or procedure type (Table 4). Mean words per sentence differed significantly between classifications for articles published by GMWs $(P=.047)$ and combined type articles $(P=.026$, Table 5$)$. Statistically significant differences between classifications were observed in mean sentences per paragraph for cervical type articles only $(P=.008$, Table 6). Statistically significant differences in FRE score were demonstrated for HI publication sources $(P=.035)$ and combined type articles $(P=.037$, Table 7$)$. Statistically significant differences in FK grade between classifications were observed for HI sources $(P=.028)$ and combined type articles $(P=.020$, Table 8).

\section{DISCUSSION}

Outpatient spine procedures are becoming an increasingly popular option globally with even greater popularity of ASCs within the United States alone. While providers may fully appreciate the benefits of this option, patients often will use 
Table 3. Mean word count for procedures and settings.

\begin{tabular}{|c|c|c|c|c|}
\hline & Total $(n=342)$ & Outpatient $(n=279)$ & Ambulatory $(n=63)$ & $P$ Value ${ }^{\mathrm{a}, \mathrm{b}}$ \\
\hline \multicolumn{5}{|l|}{ Institution } \\
\hline Hospital or institution & $733.0 \pm 562.1$ & $729.6 \pm 583.2(106)$ & $765.2 \pm 305.6(11)$ & 0.843 \\
\hline General medical & $963.4 \pm 753.2(100)$ & $922.2 \pm 695.4(68)$ & $1051.0 \pm 868.7(32)$ & 0.428 \\
\hline Private practice & $555.9 \pm 434.5(93)$ & $556.7 \pm 441.9(88)$ & $541.4 \pm 306.0$ & 0.940 \\
\hline Surgery center & $708.0 \pm 812.4(32)$ & $887.5 \pm 1050.0(17)$ & $504.5 \pm 343.5(15)$ & 0.188 \\
\hline \multicolumn{5}{|l|}{ Procedures } \\
\hline Cervical & $788.1 \pm 664.2(63)$ & $880.6 \pm 704.4$ & $395.2 \pm 151.7$ & 0.022 \\
\hline Lumbar & $719.0 \pm 545.7(84)$ & $672.8 \pm 519.4$ & $1104 \pm 637.7$ & 0.024 \\
\hline Not specified & $570.0 \pm 468.0(102)$ & $502.2 \pm 415.5$ & $768.4 \pm 558.8$ & 0.012 \\
\hline Combined & $949.0 \pm 790.2(93)$ & $916.8 \pm 748.4$ & $1104.3 \pm 980.1$ & 0.391 \\
\hline
\end{tabular}

${ }^{a} P$ value calculated using independent $t$ tests (continuous).

${ }^{\mathrm{b}}$ Boldface $\mathrm{P}$ value $(<.05)$ indicates a statistically significant difference between the two groups being compared.

Table 4. Characters per word count for procedures and settings.

\begin{tabular}{|c|c|c|c|c|}
\hline & Total $(n=342)$ & Outpatient $(n=279)$ & Ambulatory $(n=63)$ & $P$ Value $^{\mathrm{a}}$ \\
\hline \multicolumn{5}{|l|}{ Institution } \\
\hline Hospital or institution & $5.1 \pm 0.4(117)$ & $5.1 \pm 0.4$ & $5.3 \pm 0.3$ & 0.097 \\
\hline General medical & $5.2 \pm 0.3(100)$ & $5.2 \pm 0.3$ & $5.2 \pm 0.3$ & 0.368 \\
\hline Private practice & $5.1 \pm 0.3(93)$ & $5.2 \pm 0.3$ & $5.0 \pm 0.3$ & 0.270 \\
\hline Surgery center & $6.6 \pm 8.8(32)$ & $8.0 \pm 12.1$ & $5.1 \pm 0.3$ & 0.350 \\
\hline \multicolumn{5}{|l|}{ Procedures } \\
\hline Cervical & $5.1 \pm 0.3$ & $5.1 \pm 0.3$ & $5.1 \pm 0.4$ & 0.924 \\
\hline Lumbar & $5.1 \pm 0.3$ & $5.1 \pm 0.3$ & $5.1 \pm 0.2$ & 0.698 \\
\hline Not specified & $5.6 \pm 4.9$ & $5.8 \pm 5.7$ & $5.2 \pm 0.3$ & 0.572 \\
\hline Combined & $5.3 \pm 0.3$ & $5.2 \pm 0.3$ & $5.3 \pm 0.2$ & 0.330 \\
\hline
\end{tabular}

${ }^{\mathrm{a}} P$ value calculated using independent $t$ tests (continuous).

Table 5. Words per sentence for procedures and settings.

\begin{tabular}{|c|c|c|c|c|}
\hline & Total $(n=342)$ & Outpatient $(\mathrm{n}=279)$ & Ambulatory $(n=63)$ & $P$ Value $^{\mathrm{a}}$ \\
\hline \multicolumn{5}{|l|}{ Institution } \\
\hline Hospital or institution & $19.7 \pm 3.9(117)$ & $19.5 \pm 4.0$ & $21.2 \pm 2.9$ & 0.176 \\
\hline General medical & $20.6 \pm 4.3(100)$ & $20.0 \pm 4.3$ & $21.8 \pm 4.2$ & 0.047 \\
\hline Private practice & $19.7 \pm 4.3(93)$ & $19.8 \pm 4.3$ & $18.9 \pm 4.5$ & 0.650 \\
\hline Surgery center & $17.3 \pm 3.3(32)$ & $16.8 \pm 3.3$ & $17.8 \pm 3.4$ & 0.397 \\
\hline \multicolumn{5}{|l|}{ Procedures } \\
\hline Cervical & $20.7 \pm 4.8$ & $20.5 \pm 4.8$ & $21.7 \pm 5.0$ & 0.455 \\
\hline Lumbar & $18.4 \pm 3.2$ & $18.5 \pm 3.3$ & $17.6 \pm 2.2$ & 0.453 \\
\hline Not specified & $20.0 \pm 4.7$ & $19.9 \pm 4.9$ & $20.2 \pm 4.1$ & 0.752 \\
\hline Combined & $20.0 \pm 3.6$ & $19.6 \pm 3.5$ & $21.8 \pm 3.7$ & 0.026 \\
\hline
\end{tabular}

${ }^{\text {a }} P$ value calculated using independent $t$ tests (continuous).

${ }^{\mathrm{b}}$ Boldface $\mathrm{P}$ value $(<0.05)$ indicates a statistically significant difference between the two groups being compared.

Table 6. Sentences per paragraph for procedures and settings.

\begin{tabular}{|c|c|c|c|c|}
\hline & Total $(n=342)$ & Outpatient $(n=279)$ & Ambulatory $(n=63)$ & $P$ Value $^{\mathrm{a}, \mathrm{b}}$ \\
\hline \multicolumn{5}{|l|}{ Institution } \\
\hline Hospital or institution & $2.9 \pm 1.9$ & $2.9 \pm 1.6$ & $3.6 \pm 4.0$ & 0.213 \\
\hline General medical & $2.7 \pm 2.3$ & $2.8 \pm 2.7$ & $2.6 \pm 1.3$ & 0.689 \\
\hline Private practice & $2.8 \pm 1.4$ & $2.8 \pm 1.4$ & $2.6 \pm 0.7$ & 0.789 \\
\hline Surgery center & $2.6 \pm 1.2$ & $3.0 \pm 1.4$ & $2.2 \pm 0.6$ & 0.053 \\
\hline \multicolumn{5}{|l|}{ Procedures } \\
\hline Cervical & $2.6 \pm 1.2$ & $2.8 \pm 1.3$ & $1.8 \pm 0.5$ & 0.008 \\
\hline Lumbar & $2.8 \pm 1.4$ & $2.7 \pm 1.3$ & $3.5 \pm 1.9$ & 0.094 \\
\hline Not specified & $2.6 \pm 1.5$ & $2.6 \pm 1.7$ & $2.3 \pm 0.8$ & 0.317 \\
\hline Combined & $3.2 \pm 2.7$ & $3.1 \pm 2.6$ & $3.5 \pm 3.2$ & 0.671 \\
\hline
\end{tabular}

${ }^{\text {a }} P$ value calculated using independent $t$ tests (continuous).

${ }^{\mathrm{b}}$ Boldface $\mathrm{P}$ value $(<0.05)$ indicates a statistically significant difference between the two groups being compared. 
Table 7. Flesch Reading Ease for procedures and settings.

\begin{tabular}{|c|c|c|c|c|}
\hline & Total $(n=342)$ & Outpatient $(n=279)$ & Ambulatory $(n=63)$ & $P$ Value $^{\mathrm{a}, \mathrm{b}}$ \\
\hline \multicolumn{5}{|l|}{ Institution } \\
\hline Hospital or institution & $39.9 \pm 12.5$ & $40.7 \pm 12.6$ & $32.4 \pm 8.1$ & 0.035 \\
\hline General medical & $35.4 \pm 14.1$ & $36.8 \pm 14.7$ & $32.3 \pm 12.3$ & 0.140 \\
\hline Private practice & $38.0 \pm 11.6$ & $37.7 \pm 11.6$ & $43.6 \pm 11.4$ & 0.271 \\
\hline Surgery center & $41.0 \pm 13.6$ & $40.1 \pm 15.6$ & $42.0 \pm 11.4$ & 0.702 \\
\hline \multicolumn{5}{|l|}{ Procedures } \\
\hline Cervical & $39.9 \pm 13.8$ & $40.1 \pm 13.4$ & $38.9 \pm 15.8$ & 0.792 \\
\hline Lumbar & $41.2 \pm 13.6$ & $41.4 \pm 13.9$ & $39.0 \pm 11.8$ & 0.619 \\
\hline Not specified & $37.4 \pm 13.0$ & $37.8 \pm 13.6$ & $36.2 \pm 11.4$ & 0.585 \\
\hline Combined & $35.1 \pm 10.9$ & $36.2 \pm 11.0$ & $30.0 \pm 8.9$ & 0.037 \\
\hline
\end{tabular}

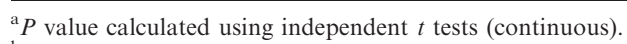

${ }^{\mathrm{b}}$ Boldface $\mathrm{P}$ value $(<0.05)$ indicates a statistically significant difference between the two groups being compared.

sources beyond the clinician to comprehend surgical choices. Although hospitals, journals, institutions, and other literature sources may attempt to generate readable information through their respective online sources, the responsibility of understanding the literature falls solely on the patient. As many spine procedures increasingly trend toward the use of ASCs, ensuring the relevant information provided to patients is at an appropriate level for comprehension becomes essential. Authors of other studies have assessed the readability of content related to fusion procedures, spine surgery, and minimally invasive surgery; however, in this study, we aimed to investigate literature pertaining to outpatient hospital settings and ASCs. We were able to determine that information regarding outpatient hospitals and ASCs were significantly different to comprehend when published from HIs, especially when it pertained to topics that included cervical and lumbar procedures.

In total, we were able to review 279 outpatient and 63 ambulatory center articles and demonstrated an overall nonsignificant difference between FRE scores and FK grade levels. Although no significant difference was observed, the level with which the scores and grade correspond can be categorized as both difficult to read and at college reading level, which is far beyond the recommended level. Comparison with past studies proved difficult as many investigators dissimilarly categorized sources of information; however, academic, organizational, and hospital were common sources. Our readability scores from HI sources reported a mean FRE score of 40.7 for outpatient and 32.4 for ambulatory settings, which are similarly reported in minimally invasive and open spinal surgery literature. ${ }^{20}$ Although our topics differed from previous studies, other investigators found that readability scores of spine-surgery-related topics from top academic institutions and hospitals in the United States had an overall range of FRE scores of 11.0 to $11.8,{ }^{18}$ and authors of sources similar to our study reported a mean of $10.9,{ }^{19}$ both substantially lower than our findings. This may suggest that, although ASCs and outpatient hospital settings may fall under spinesurgery-related topics, it may be a subject patients could better comprehend. However, it should be noted that, while the mean FRE scores may have drastically different numbers, a more in-depth comparison of the 2 topics may be required to further elucidate which areas of spine surgery contribute to a lower score than outpatient or ASC topics. Additionally, it is interesting to note that comparison of outpatient-related to ASC-

Table 8. Flesch-Kincaid grade for procedures and settings.

\begin{tabular}{|c|c|c|c|c|}
\hline & Total $(n=342)$ & Outpatient $(n=279)$ & Ambulatory $(n=63)$ & $P$ Value ${ }^{\mathrm{a}, \mathrm{b}}$ \\
\hline \multicolumn{5}{|l|}{ Institution } \\
\hline Hospital or institution & $12.5 \pm 2.3$ & $12.3 \pm 2.4$ & $13.9 \pm 1.2$ & 0.028 \\
\hline General medical & $13.2 \pm 2.9$ & $13.0 \pm 2.8$ & $13.7 \pm 3.0$ & 0.280 \\
\hline Private practice & $12.8 \pm 2.9$ & $12.8 \pm 2.9$ & $11.8 \pm 2.2$ & 0.446 \\
\hline Surgery center & $11.8 \pm 2.1$ & $11.8 \pm 2.4$ & $11.8 \pm 1.8$ & 0.972 \\
\hline \multicolumn{5}{|l|}{ Procedures } \\
\hline Cervical & $12.4 \pm 3.2$ & $12.4 \pm 3.1$ & $12.3 \pm 3.8$ & 0.888 \\
\hline Lumbar & $12.0 \pm 2.5$ & $12.0 \pm 2.5$ & $12.1 \pm 1.9$ & 0.931 \\
\hline Not specified & $13.0 \pm 2.8$ & $13.0 \pm 3.0$ & $13.1 \pm 2.3$ & 0.802 \\
\hline Combined & $13.2 \pm 2.1$ & $13.0 \pm 2.1$ & $14.3 \pm 1.8$ & 0.020 \\
\hline
\end{tabular}

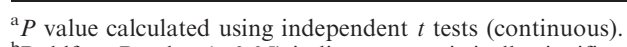

${ }^{\mathrm{b}}$ Boldface $\mathrm{P}$ value $(<0.05)$ indicates a statistically significant difference between the two groups being compared. 
related literature demonstrated a significant difference, with outpatient articles having a higher FRE score and lower FK grade level, which suggests that literature regarding outpatient hospital settings may currently be better suited for informing patients than ASC literature.

While we were able to determine a significant difference in the ease of readability between outpatient- and ambulatory-related articles produced by HIs, there were no significant differences between the articles being produced by general medical sources, PPs, and SCs. In this study, we found that the readability of both outpatient and ambulatory centers were noticeably better than topics such as spinal fusion or spine procedures in general. ${ }^{19}$ This observation is not surprising, given the topics of spine surgery that may require more technical explanations than explanation of an outpatient setting. More interestingly, the significant difference in FK grade and FRE scores by group was demonstrated when articles pertained to combined procedures (cervical and lumbar) rather than only cervical or lumbar topics. The respective FRE scores were again noticeably higher than previous studies. ${ }^{18,19,22}$

While, in our study, we showcased the increased ease of readability with regard to ambulatory- or outpatient-related articles compared with other topics in spine surgery, their respective scores, regardless of topic or source, remained approximately 6 grades above the level recommended for patient-targeted medical literature by the National Institutes of Health, American Medical Association, and U.S. Department of Health and Human Resources. This observation is not unique to our study as several previous readability studies have reported the inappropriate reading level of spinerelated material. ${ }^{18-21,23}$ To avoid reading level inappropriateness, guidelines offered by national or local health agencies, such as the Centers for Disease Control and Prevention (CDC), can provide checklists and standardized instructions on composing health-related material. As an example, the CDC's guidelines help standardize areas such as message content, text appearance, visuals, layout design, translation (if necessary), and understandability. ${ }^{15}$

In addition to standardizing reading material, alternative forms of health information delivery should be considered. Video platforms may be an appropriate alternative, as authors of previous studies in colonoscopy patients demonstrated a significant increase in quality of bowel preparation among patients receiving a brief preprocedural instructional video. ${ }^{24}$ However, the source of video again must be standardized, as a number of investigators have reported their concern with use of common video platforms such as YouTube, which may offer low-quality information. ${ }^{25-27}$

\section{Limitations}

This study is not without several limitations. Readability was assessed using the FRE score and FK grade level; however, many other scores exist which may provide additional insight into the difficulty patients may experience with outpatient or ambulatory literature. Additionally, the FRE score and FK grade have their own limitations. More specifically, the score ranges from 0 to 100 and is difficult to interpret, and the grade level may underestimate or overestimate the readability of medical terminology due to its evaluation being based on polysyllabic words. ${ }^{28}$ The method used to collect relevant articles may have also limited our study. Given searches were performed through online general search engines and not through a standardized database such as Medline or Embase, the sources of information vary widely in terms of quality of content, region, and standards of the outpatient or ASC. Another limitation is not including pictures or infographic-based material which may provide increased readability and comprehension. One aspect that should also be considered as a limitation of the study is the exclusion of non-English articles as well as not identifying the country of origin of each article. This may introduce some bias toward the information provided to patients in English-speaking countries only.

\section{CONCLUSIONS}

Literature found on the Internet with regard to outpatient and ASCs is found to be written at a reading level that is much higher than an average English-speaking adult can comprehend. This trend is like previous studies and reinforces the notion that a concerted effort should be made to write online material that better conveys the use of both ASCs and outpatient hospital settings for surgical treatment. As the use of ASCs or outpatient hospital settings continues to grow, patients will 
consistently rely on the Internet as one of their main resources of information. Without change, there will be a continued disconnect between what the patient comprehends and what the provider believes the patient comprehends. To produce effective patient information, a reduction in the number of words per sentence and syllables per sentence would generate improved reading ease. Additionally, authors should consider the use of more standardized practices in the delivery of health-related information according to health agency guidelines and focus on the differences in the surgical settings alone rather than including complex topics such as surgical techniques. Future studies should address this continued gap in readability and determine if material that achieves the recommended reading level for an English-speaking adult has an impact on the patient's comprehension of both the type and place of treatment.

\section{REFERENCES}

1. Idowu OA, Boyajian HH, Ramos E, Shi LL, Lee MJ. Trend of spine surgeries in the outpatient hospital setting versus ambulatory surgical center. Spine (Phila Pa 1976). 2017;42(24):E1429-E1436.

2. Arshi A, Wang C, Park HY, et al. Ambulatory anterior cervical discectomy and fusion is associated with a higher risk of revision surgery and perioperative complications: an analysis of a large nationwide database. Spine J. 2018;18(7):1180-1187.

3. Chin KR, Pencle FJR, Coombs AV, Packer CF, Hothem EA, Seale JA. Eligibility of outpatient spine surgery candidates in a single private practice. Clin Spine Surg. 2017;30(10):E1352E1358.

4. Patel DV, Yoo JS, Haws BE, et al. Comparative analysis of anterior cervical discectomy and fusion in the inpatient versus outpatient surgical setting. J Neurosurg Spine. 2019;1-6. doi:10.3171/2019.1.SPINE181311

5. Patel DV, Yoo JS, Karmarkar SS, Lamoutte EH, Singh $\mathrm{K}$. Minimally invasive lumbar decompression in an ambulatory surgery center. J Spine Surg. 2019;5(Suppl 2):S166-S173.

6. Bekelis K, Missios S, Kakoulides G, Rahmani R, Simmons N. Selection of patients for ambulatory lumbar discectomy: results from four US states. Spine $J$. 2014;14(9):1944-1950.

7. Helseth Ø, Lied B, Halvorsen CM, Ekseth K, Helseth E. Outpatient cervical and lumbar spine surgery is feasible and safe: a consecutive single center series of 1449 patients. Neurosurgery. 2015;76(6):728-737; discussion 737-738.

8. Sivaganesan A, Hirsch B, Phillips FM, McGirt MJ. Spine surgery in the ambulatory surgery center setting: value-based advancement or safety liability? Neurosurgery. 2018;83(2):159165.

9. Liu JT, Briner RP, Friedman JA. Comparison of inpatient vs. outpatient anterior cervical discectomy and fusion: a retrospective case series. BMC Surg. 2009;9:3. doi:10.1186/ 1471-2482-9-3

10. Ortega A, Sarmiento JM, Patil C, et al. Comparative analysis of inpatient and outpatient interspinous process device placement for lumbar spinal stenosis. J Neurol Surg A Cent Eur Neurosurg. 2015;76(6):443-450.

11. Mullins J, Pojskić M, Boop FA, Arnautović KI. Retrospective single-surgeon study of 1123 consecutive cases of anterior cervical discectomy and fusion: a comparison of clinical outcome parameters, complication rates, and costs between outpatient and inpatient surgery groups, with a literature review. J Neurosurg Spine. 2018;28(6):630-641.

12. Liebner LT. I can't read that! Improving perioperative literacy for ambulatory surgical patients. AORN J. 2015;101(4):416-427.

13. Kutner M, Greenberg E, Jin Y, Paulsen C. The Health Literacy of America's Adults: Results from the 2003 National Assessment of Adult Literacy. National Center for Education Statistics; 2006. doi:10.1037/e530912012-001

14. Cotugna N, Vickery CE, Carpenter-Haefele KM. Evaluation of literacy level of patient education pages in health-related journals. J Community Health. 2005;30(3):213219.

15. Centers for Disease Control and Prevention. Simply Put: A Guide for Creating Easy-to-Understand Materials. CDC Atlanta; 2009.

16. Institute of Medicine, Board on Neuroscience and Behavioral Health, Committee on Health Literacy. Health Literacy: A Prescription to End Confusion. National Academies Press; 2004.

17. Fields AM, Freiberg CS, Fickenscher A, Shelley KH. Patients and jargon: are we speaking the same language? J Clin Anesth. 2008;20(5):343-346.

18. Ryu JH, Yi PH. Readability of spine-related patient education materials from leading orthopedic academic centers. Spine (Phila Pa 1976). 2016;41(9):E561-E565.

19. Long WW, Modi KD, Haws BE, et al. Assessing online patient education readability for spine surgery procedures. Clin Spine Surg. 2018;31(2):E146-E151.

20. Agarwal N, Feghhi DP, Gupta R, et al. A comparative analysis of minimally invasive and open spine surgery patient education resources. J Neurosurg Spine. 2014;21(3):468-474.

21. Zhang D, Schumacher C, Harris MB, Bono CM. The quality and readability of information available on the Internet regarding lumbar fusion. Global Spine J. 2016;6(2):133-138.

22. Vives M, Young L, Sabharwal S. Readability of spinerelated patient education materials from subspecialty organization and spine practitioner Websites. Spine (Phila Pa 1976). 2009;34(25):2826-2831.

23. Eltorai AEM, Cheatham M, Naqvi SS, et al. Is the readability of spine-related patient education material improving? An assessment of subspecialty Websites. Spine (Phila Pa 1976). 2016;41(12):1041-1048

24. Prakash SR, Verma S, McGowan J, et al. Improving the quality of colonoscopy bowel preparation using an educational video. Can J Gastroenterol. 2013;27(12):696-700.

25. Erdem MN, Karaca S. Evaluating the accuracy and quality of the information in kyphosis videos shared on YouTube. Spine (Phila Pa 1976). 2018;43(22):E1334-E1339.

26. Ovenden CD, Brooks FM. Anterior cervical discectomy and fusion YouTube videos as a source of patient education. Asian Spine J. 2018;12(6):987-991.

27. Brooks FM, Lawrence H, Jones A, McCarthy MJH. YouTube $^{\mathrm{TM}}$ as a source of patient information for lumbar discectomy. Ann R Coll Surg Engl. 2014;96(2):144-146. 
28. Friedman DB, Hoffman-Goetz L. A systematic review of readability and comprehension instruments used for print and Web-based cancer information. Health Educ Behav. 2006;33(3):352-373.

Disclosures and COI: No funds were received in support of this work. No benefits in any form have been or will be received from any commercial party related directly or indirectly to the subject of this manuscript.

Corresponding Author: Kern Singh, MD, Department of Orthopaedic Surgery, Rush Univer- sity Medical Center, 1611 W. Harrison St, Suite \#300, Chicago, IL 60612. Phone: (312) 432-2373; Fax: (708) 409-5179; Email: kern.singh@rushortho. com.

Published 3 December 2021

This manuscript is generously published free of charge by ISASS, the International Society for the Advancement of Spine Surgery. Copyright (C) 2021 ISASS. To see more or order reprints or permissions, see http://ijssurgery.com. 